\title{
Modeling Microscopic Chemical Sensors in Capillaries
}

\author{
Tad Hogg* \\ Hewlett-Packard Laboratories, Palo Alto, CA, USA
}

\begin{abstract}
Nanotechnology-based microscopic robots could provide accurate in vivo measurement of chemicals in the bloodstream for detailed biological research and as an aid to medical treatment. Quantitative performance estimates of such devices require models of how chemicals in the blood diffuse to the devices. This paper models microscopic robots and red blood cells (erythrocytes) in capillaries using realistic distorted cell shapes. The models evaluate two sensing scenarios: robots moving with the cells past a chemical source on the vessel wall, and robots attached to the wall for longer-term chemical monitoring of chemicals released into the bloodstream. Using axial symmetric geometry with realistic flow speeds and diffusion coefficients, we compare detection performance with a simpler model that does not include the cells. The average chemical absorption is quantitatively similar in both models, indicating the simpler model is an adequate design guide to sensor performance in capillaries. However, determining the variation in forces and absorption as cells move requires the full model.
\end{abstract}

\section{INTRODUCTION}

Nanotechnology has the potential to greatly improve health care [1-3]. For example, nanoscale particles can significantly enhance medical imaging [4] and drug delivery [5]. Future possibilities include programmable machines comparable in size to cells and able to sense and modify their environments. Such microscopic robots could provide significant medical benefits $[1,6]$ with precisely controlled targeted actions at the scale of individual cells.

While microscopic robots cannot yet be manufactured, current laboratory demonstrations indicate their likely minimal capabilities based on nanoscale electronics, sensors and motors [7-13]. These capabilities and estimates of the properties of in vivo environments where the devices would operate are sufficient to develop computational models of device performance for a variety of medical tasks.

A particularly important robot environment is the blood vasculature, which allows the robots ready access throughout the body via passive movement with the fluid. A challenge for computational models of devices in blood is the interactions among the blood components and the devices. The blood is not a uniform fluid on the scale of the devices but rather consists of larger objects (the cells) embedded in a fluid (the plasma). Thus detailed models must include fluid dynamics with cells that move and distort, branching vessels, changes in vessel size, and hydrodynamic interactions among the devices. Of particular interest is the behavior of microscopic devices in capillaries, which exchange chemicals with body tissues and bring the devices close to cells throughout the body.

Detailed models including numerous cells interacting with the devices are computationally intensive. Thus design studies of medical task scenarios benefit from simpler

*Address correspondence to this author at the Hewlett-Packard Laboratories, Palo Alto, CA, USA; E-mail: tad.hogg@hp.com models that nevertheless give reasonable quantitative estimates of task performance. One such model considers the blood as a homogeneous fluid, with possibly modified parameters, at the scale of the robots. One important process to model is transport of chemicals to the devices, which is similar to the process for bacteria [14]. Chemical transport is key for medical tasks such as high-resolution diagnosis [15], treatment through targeted drug delivery [5, 16-18], microsurgery within and among individual cells $[6,19,20]$ and power generation using chemicals available in the bloodstream. An example of power generation in the robots is combining glucose and oxygen in fuel cells [6] analogous to the enzyme-mediated reactions in bacteria [21].

One modeling approximation, suited to vessels wide enough to accommodate multiple cells across their diameter, treats the effect of cells as increasing diffusion in vessels large enough to allow cells to rotate [22]. In this case, a model with homogeneous fluid and an increase in the chemical diffusion coefficients gives a reasonable approximation to chemical transport in the vessel. However, in capillaries cells move through single file so this approach is not suitable.

As an aid to modeling behavior in capillaries, this paper compares a simple model (homogeneous fluid with no cells) with the effect of cells moving in the fluid. The evaluation of behavior with cells is simplified to use realistic but approximate geometry for deformed cell shape based on prior numerical studies of mechanical properties of cells and their membranes. This approach avoids the computational cost of simultaneously solving for the shape of the cell due to interaction with fluid, while still being useful to calibrate models not including cells. The models considered here are similar to previous studies of chemical transport in blood vessels [23], but with scenarios relevant for microscopic robots. In particular, the robots considered here, of size comparable to bacteria, are somewhat smaller than blood and tissue cells, and so can involve steeper concentration gradients. In particular, this model captures two key effects 
of cells in capillaries: their effect on the fluid flow and their restriction on chemical diffusion within the plasma. The remainder of the paper describes the cell model and compares its predictions with the simpler homogeneous fluid model.

\section{METHOD}

We examine the effect of cells on microscopic chemical sensors in capillaries by numerically evaluating the fluid flow and chemical diffusion. We consider a cylindrical vessel [24] and an axial-symmetric geometry with the cells centered along the axis of the vessel. Fluid motion in these small vessels is dominated by viscosity. Such microfluidic flows are generally smooth and laminar with little mixing [25], leading to physical behaviors distinct from those familiar with macroscopic flows [26, 27].

Applications for microscopic devices involve both small and large molecules dissolved in the fluid. The diffusion coefficients for such molecules in fluids at body temperature range from $2 \times 10^{-9} \mathrm{~m}^{2} / \mathrm{s}$ for small molecules (e.g., oxygen and carbon dioxide) to around $10^{-10} \mathrm{~m}^{2} / \mathrm{s}$ for a typical 10 kilodalton protein [6]. We examine behavior for both these diffusion coefficients and a range of typical flow speeds in capillaries. Table $\mathbf{1}$ gives the model parameters for the vessel, fluid properties, cell geometry and chemical diffusion.

Table 1. Model Parameters. The volume and surface area are typical values for red blood cells [28]. The vessel size and hematocrit (fraction of vessel volume occupied by cells) are typical for capillaries, and fluid density and viscosity for plasma are similar to the values of water at body temperature [6]

\begin{tabular}{|l|l|}
\hline Parameter & Value \\
\hline \hline vessel radius & $R=3 \mu \mathrm{m}$ \\
fluid density & $\rho=10^{3} \mathrm{~kg} / \mathrm{m}^{3}$ \\
fluid viscosity & $\eta=10^{-3} \mathrm{~Pa} \cdot \mathrm{s}$ \\
hematocrit & $h=25 \%$ \\
cell speed & $v_{\text {cell }}=0.2,1$ and $2 \mathrm{~mm} / \mathrm{s}$ \\
\hline \multicolumn{2}{|c|}{ cell geometry } \\
cell volume & $V=90 \mu \mathrm{m}^{3}$ \\
cell surface area & $S=135 \mu \mathrm{m}^{2}$ \\
cell spacing & $L=V /\left(\pi R^{2} h\right)=12.7 \mu \mathrm{m}$ \\
\hline \multicolumn{2}{|c|}{ diffusion coefficients } \\
small molecules & $D_{\text {small }}=2 \times 10^{-9} \mathrm{~m}^{2} / \mathrm{s}$ \\
large molecules & $D_{\text {large }}=10^{-10} \mathrm{~m}^{2} / \mathrm{s}$ \\
\hline
\end{tabular}

\section{Cell Geometry}

Unlike the biconcave shape of red blood cells at rest, cells moving through narrow vessels are considerably distorted into a bullet-like shape [28, 29]. Nevertheless, the cells maintain their volume and surface area since their contents are nearly incompressible and the membrane strongly resists changes in area [30]. More complex interactions between cells occur where vessels bend or branch [31].

Significantly for chemical transport through the plasma to sensors, the flow establishes a gap between the distorted cell and the vessel wall. The gap between the cell and vessel wall depends on the speed of the cell in the vessel, as indicated in Table 2 for a vessel of radius $R=3 \mu \mathrm{m}$. As flow speed increases, the gap increases and cells become narrower and longer.

Table 2. Gap between Cell and Vessel Wall as a Function of Cell Speed, $v_{\text {cell }}$, in a Capillary with Radius of Three Microns [28]

\begin{tabular}{|l|ccc|}
\hline cell speed $(\mathrm{mm} / \mathrm{s})$ & 0.2 & 1 & 2 \\
\hline $\operatorname{gap}(\mu \mathrm{m})$ & 0.7 & 0.9 & 1.0 \\
\hline
\end{tabular}

To estimate the effect on chemicals reaching microscopic sensors, we consider an approximate geometry for the cells including most of the distortion from the flow. For a vessel of uniform diameter far from its merge with other vessels, we consider the cells as achieving their equilibrium shape with respect to the flow and avoid explicitly modeling the forces distorting the cell.

We describe each cell as a solid of revolution defined by the curve illustrated in Fig. (1). This curve consists of parts of three circles and a line segment. The curve is continuous and smooth, i.e., the parts match with continuous first derivative. Specifically, the front of the cell model consists of a quarter-circle, with radius $r$, connected to a straight line parallel to the vessel axis of length $a$. The back of this line connects to a small half-circle which connects to another quarter-circle, of radius $s$, forming the trailing edge of the cell model. The full 3-dimensional form of the cell consists of rotating the area enclosed by these curves around the vessel axis, giving an axially symmetric cell shape. This geometry, similar to that employed in some models of oxygen transport [32], approximates shapes obtained through numerical evaluation of the fluid forces on the cells [28, 29].

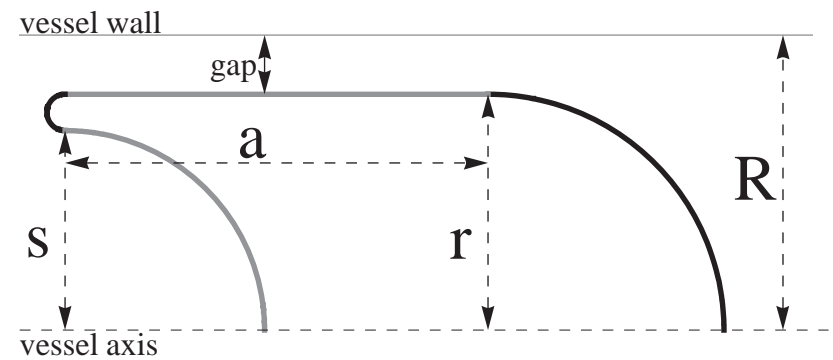

Fig. (1). Cell geometry showing a cross section of half the vessel, from the axis on the bottom to the vessel wall at the top. The model involves 4 joined curves, shown as alternating black and gray parts. The cell moves from left to right through the vessel.

We determine the three geometric parameters of the cell model, $r, a$ and $s$, from three constraints. First, we pick $R-$ $r$ to equal the gap between the cell and vessel wall given in Table 2, where $R$ is the vessel radius. Second, we pick $a$ and $s$ so the cell volume and surface area for the 3-dimensional shape match the values in Table 1. Fig. (2) shows the 
resulting cell model shapes for various speeds of the cells through the vessel.

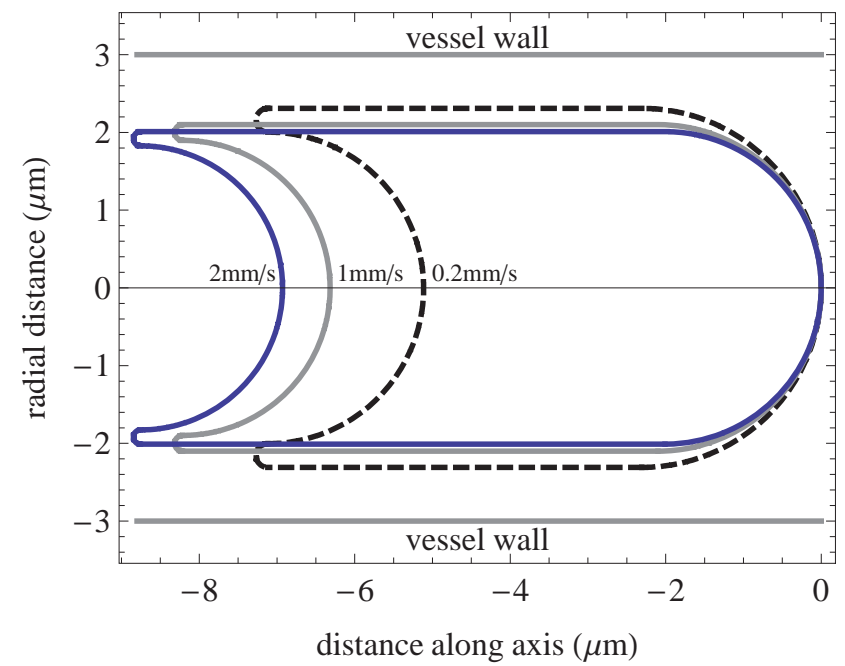

Fig. (2). Cell geometries for speeds $v_{\text {cell }}=0.2,1$ and $2 \mathrm{~mm} / \mathrm{s}$. The figure shows a cross section through the vessel, centered on the vessel axis. The front of each cell is shown in the same location along the vessel, at the right. The cell and fluid move from left to right.

Cells pass in single file through the capillary. For simplicity, we consider the cells to all have the same size and to be uniformly spaced. The spacing between cells is determined by the hematocrit value, $h$, in capillaries, i.e., the fraction of volume occupied by red blood cells. The hematocrit in capillaries is generally somewhat smaller than in larger vessels. With distance $L$ between the fronts of successive cells, as shown in Fig. (3), the cells occupy a fraction $V /\left(\pi R^{2} L\right)$ of the vessel volume, which corresponds to the hematocrit $h$. Equating these values gives the value of $L$ in Table 1.

\section{Chemical Sensor}

For the chemical sensor, we consider two scenarios, with parameters given in Table $\mathbf{3}$.

In the first scenario, appropriate for long term monitoring, the sensor is a group of robots forming a uniform band embedded in the vessel wall [6]. This band geometry maintains axial symmetry. In this scenario, we suppose a chemical is released into the vessel well upstream of the sensor band. Eventually the fluid brings the chemical past the sensor band. Thus the chemical of interest is in the plasma surrounding the cells in the vessel and is brought past the sensor by the blood flow. The main performance question is how much of the chemical in the fluid reaches the sensor for capture rather than being driven past the sensor by the fluid flow.

Table 3. Parameters for Two Sensing Scenarios. The sensor size is a design choice commonly considered in studies of microscopic sensors [6, 33], and small enough to pass through even the smallest blood vessels. The source length considered here, for a chemical source on the vessel wall, is comparable to the size of a single cell which provides a challenging detection task for microscopic sensors. The choice of inlet concentration and source flux are discussed in the text.

\begin{tabular}{|l|l|}
\hline Parameter & Value \\
\hline \hline sensor length & $\ell=2 \mu \mathrm{m}$ \\
scenario 1: sensor band on vessel wall \\
inlet concentration & $C=10^{17}$ molecule $/ \mathrm{m}^{3}$ \\
scenario 2: sensor moving with fluid \\
source length & $\lambda=10 \mu \mathrm{m}$ \\
source flux & $K=10^{13}$ molecule $/ \mathrm{s} / \mathrm{m}^{2}$ \\
\hline
\end{tabular}

The band of sensors around the vessel wall could consist of multiple rings of devices, but for simplicity we consider the most challenging case for chemical detection: the smallest possible size consistent with axial symmetry of a single device length along the length of the vessel, i.e., the band has length $\ell$ along the vessel and consists of about $2 \pi R / \ell \approx 9$ individual sensors forming the band around the vessel. The sensor band has a total luminal surface area of $2 \pi R \ell=38 \mu \mathrm{m}^{2}$.

Such a sensor band could most readily be constructed from a collection of microscopic devices moving through the circulation and aggregating at a suitable location, e.g., marked by a pattern of chemicals of interest [33]. In this way, individual devices could be small enough to flow through even the smallest capillaries and build a larger aggregate structure on the vessel wall [6].

In the second sensing scenario, we consider a single sensor flowing in the vessel between two cells and the chemical source is a single cell-sized region on the vessel wall. In this case we take the sensor to be spherical with diameter $\ell$, and moving in the center of the vessel, thereby

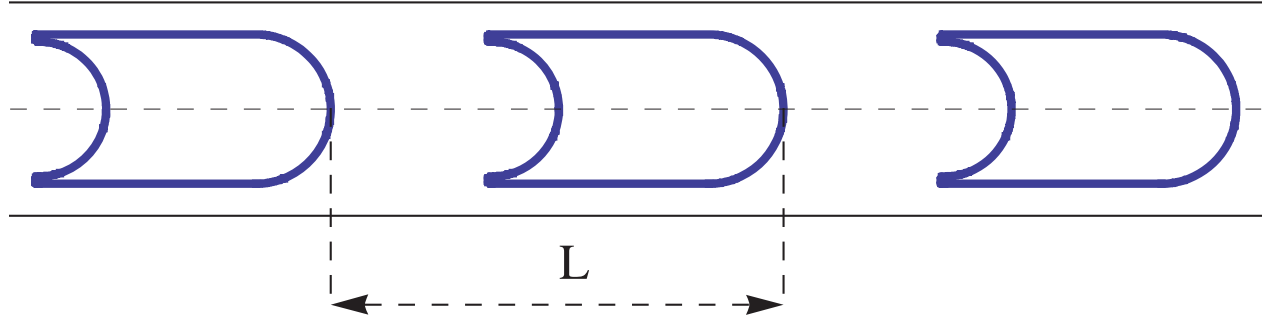

Fig. (3). Cell spacing, using the geometry for speed of $1 \mathrm{~mm} / \mathrm{s}$. The figure shows a cross section through the vessel, centered on the vessel axis. The distance between the front of successive cells is $L$. Cells and fluid move from left to right through the vessel. The upper and lower lines represent the vessel wall. The inlet and outlet boundaries are at the left and right edges of the diagram, respectively, for a finite section of a conceptually infinitely long vessel. 
maintaining axial symmetry. The specific choice of positioning of the sensor, half-way between the two cells, can be viewed either as an approximation of where it would be moved by the fluid, or as the device having active locomotion to maintain this position with respect to the cells. This model assumes the presence of the sensor makes negligible changes in the shape or positioning of the nearby cells.

This scenario corresponds to robots patrolling the circulation for chemical events of interest. Thus the performance measure is not only how much chemical the sensor detects but also how much it detects while close to the source. That is, the patrolling function can involve not only detecting the existence of a chemical source but also locating it for possible subsequent operations, such as moving to the source location [33] and releasing drugs at this location [16, 17]. To maintain axial symmetry, we take the source region to be a band around the vessel of length $\lambda=10 \mu \mathrm{m}$, thereby testing how quickly the passing sensor can detect the fairly steep concentration gradient produced by chemicals released by a single cell. We take the chemical flux to be uniform throughout this band.

A remaining question for modeling the chemical transport is whether the chemicals of interest can pass through the cell membrane (e.g., oxygen). As a specific comparison, this paper considers chemicals that do not pass through the cell membrane so their transport relevant for the sensors takes place only in the plasma. In this case the cells introduce two changes to the empty vessel model: changing the fluid flow and confining the chemical to the plasma. In contrast, a model with the chemical passing freely through the cell membranes and interiors would only affect the transport through the change in fluid flow. The numerical model examined here could readily be extended to treat more complex situations such as different transport properties (e.g., diffusion coefficients) within the cells and reactions creating or removing chemicals in the plasma or cell interiors.

Specific numerical values for the model require specifying the overall chemical concentration. The amount of a chemical in a milliliter of blood plasma relevant for medical diagnostics range from picograms for some proteins to milligrams (e.g., for glucose) [6, 34]. High resolution diagnostics for which microscopic robots would be useful involve the lower concentrations, in particular with robots passing close to the sources of rare chemicals. Near these sources, local concentrations are considerably larger than these measured values in blood samples, which correspond to the chemical diluted through-out the blood volume. For example, suppose a small source released a chemical into a few nearby capillaries. After mixing throughout the full 5liter blood volume of an adult, the concentration in a typical capillary, of length about a millimeter, dilutes by about a factor of $10^{11}$.

While this wide range of concentrations is significant for the performance of the sensors [15], the range does not affect the comparison between models with and without cells. This is because the flux to the sensor depends linearly on the concentration as specified by a boundary condition, namely the inlet concentration $C$ and source flux $K$ in the first and second scenarios, respectively. Nevertheless, for definiteness, we choose specific values for $C$ and $K$ corresponding to a typical high-resolution diagnostic task. Other situations, with either higher or lower concentrations, simply proportionally change the sensor flux values reported below. As a definite value for the first scenario, we consider a 10-kilodalton protein with concentration $2 \times 10^{-9} \mathrm{~g} / \mathrm{cm}^{3}$, which corresponds to $C=10^{17}$ molecule $/ \mathrm{m}^{3}$. A corresponding flux from a single cell producing this concentration in a nearby capillary is around $K=10^{13}$ molecule $/ \mathrm{s} / \mathrm{m}^{2}$, which gives a challenging diagnostic task [33]. For a vascular cell with luminal surface area $300 \mu \mathrm{m}^{2}$, this value corresponds to producing 3000 molecule/s, or $5 \times$ $10^{-17} \mathrm{~g} / \mathrm{s}$, through the cell membrane. We use these values for the two scenarios, summarized in Table 3.

\section{Numerical Method}

With the specified geometry of cells and sensors, we solve the partial differential equations for fluid flow and chemical transport numerically using the finite element method [35]. For numerical solution we model a segment of the vessel, as illustrated in Fig. (3) except using 10 and 20 cells in the models for the first and second scenario, respectively. In the second scenario, the model also includes the spherical sensor half-way between the middle two cells.

The Navier-Stokes equation governs the fluid flow [36]. For a periodic array of cells shown in Fig. (3) we specify the speed $v_{\text {cell }}$ at which the cells move through the vessel. A convenient numerical method is using a frame of reference moving with the cells [32, 37], analogous to the view from a camera moving at speed $v_{\text {cell }}$. In this moving reference frame, the cells are stationary while the vessel wall moves backwards. This means the fluid flow does not change with time and we compute a steady-state solution for the flow throughout the vessel in the moving reference frame.

The fluid boundary conditions are zero velocity ("no slip") on the boundaries of the cells and moving backwards with speed $-v_{\text {cell }}$ along the vessel wall. As part of the numerical solution, we find the pressure difference giving no net force on the cell in the center of the modeled vessel region. This pressure difference corresponds to the fluid pushing the cells through the vessel with a steady speed $v_{\text {cell }}$.

For chemicals in the fluid, the concentration $c$ is governed by the diffusion equation [38]

$\frac{\partial c}{\partial t}=-\nabla \cdot \mathbf{F}$

where $\mathbf{F}=-D \nabla c+\mathbf{v} c$ is the chemical flux, i.e., the rate at which molecules pass through a unit area, and $\mathbf{v}$ is the fluid velocity vector determined from the solution of the NavierStokes equation. The first term in the flux is diffusion, which acts to reduce concentration gradients, and the second term is motion of the chemical due to the movement of the fluid in which the chemical is dissolved.

As boundary conditions for the diffusion equation, we take the cells and vessel wall to be insulating, i.e., no chemical crosses those boundaries. Sufficiently far from the sensor, diffusion makes the concentration uniform throughout the vessel. In our case, far upstream from the sensor we have a known concentration at the inlet to the modeled vessel segment, described below for the two 
scenarios. Far downstream, the concentration is again uniform but its value depends on how much of the chemical is absorbed by the sensor. Thus the downstream concentration is not known a priori, and we use instead a convective boundary condition at the outlet, i.e., taking $\nabla c$ $=0$, corresponding to a uniform concentration throughout the vessel far downstream of the sensor.

The boundary conditions for the sensor and vessel inlet depend on the scenario, as does the initial condition for concentration in the vessel.

A natural boundary condition for the sensor is zero concentration, corresponding to a completely absorbing sensor. This gives the maximum rate the sensor could detect chemicals and is suitable for low concentration conditions where the sensor is diffusion-limited [39]. This will generally be the situation for detecting chemicals with low concentrations. We use this zero-concentration boundary condition for the second scenario, with the sensor moving with the fluid.

However in the first scenario, with the sensor band moving backwards with the wall, instead of a zero concentration boundary condition for the sensor and a zero flux condition on the rest of the wall, a simpler numerical approach is a time-dependent flux boundary condition for the entire wall, shown in Fig. (4). That is, at time $t$, when the sensor has moved a distance $-v_{\text {cell }} t$ from its initial location on the wall, we take flux to be $k c$ along the sensor and zero elsewhere on the wall, smoothed over a short distance, 0.2 $\mu \mathrm{m}$, to avoid discontinuity in the numerical solution. We pick $k$ large enough so the concentration along the sensor is very small, but not so large as to introduce numerical instabilities. In our case, $k=1 \mathrm{~m} / \mathrm{s}$ is sufficient to make the concentration at the sensor less than $10^{-4} C$ where $C$ is the inlet concentration. Thus this procedure approximately models a fully absorbing sensor.

In the first scenario, we model the flow bringing the chemical to the sensor from the upstream source with an initial zero concentration in the vessel segment near the source and a constant concentration $C$ at the inlet. We numerically solve the model from this initial state until the flux reaching the vessel outlet stabilizes. At this point the flux reaching the sensor becomes nearly periodic in time, with period $L / v_{\text {cell }}$ corresponding to the passage of one cell.
In the second scenario, we take the chemical source band on the vessel wall to produce the chemical with a uniform flux $K$. We solve this model in the reference frame moving with the cells, so the source on the wall moves backwards past the cells and sensor. As illustrated in Fig. (4), we model this behavior with a time-dependent boundary condition with flux $K$ at the location of the source and zero elsewhere on the vessel wall, smoothed over a short distance, $0.2 \mu \mathrm{m}$. The inlet has zero concentration.

For the initial concentration, when the source is well downstream of the sensor, all the chemical released by the source is swept downstream. Thus the concentration upstream of the source is zero and downstream the flow must contain the entire production of the source. The source produces chemical at a rate $2 \pi R \lambda K$. If the concentration far downstream is $C$, all of which is in the plasma, which occupies volume fraction $1-h$ of the vessel, then the fluid moves chemical at a rate $C \pi R^{2} v_{\text {cell }}(1-h)$. Equating these expressions gives the initial downstream concentration of $C$ $=2 \lambda K /\left(R v_{\text {cell }}(1-h)\right)$. In the simpler model without cells, the plasma fills the whole vessel so $h=0$ in this expression for the downstream concentration in that case. As initial condition, we take a smooth transition, over a distance of $10 \mu \mathrm{m}$, between 0 and this value of $C$ at the initial location of the source. We start the numerical solution with the source well downstream of the sensor, so the precise form of this initial concentration has little effect on the behavior of the sensor by the time it passes the source.

\section{RESULTS}

We compare the sensor performance in the two scenarios for the models with and without cells using the parameters of Table 1. The numerical solutions give the rate the sensors absorb the chemical. For the evenly spaced cells with identical geometry considered here, the fluid flow is independent of time and periodic, with period $L$, along the length of the vessel. In the reference frame moving with the cells, the fluid circulates between the cells, moving away from the front of the cell along the vessel axis and toward the front of the cell near the vessel wall.

\section{Sensor Band on Vessel Wall}

In the first scenario, the chemical continually enters the vessel inlet and some reaches the sensor on the wall. The

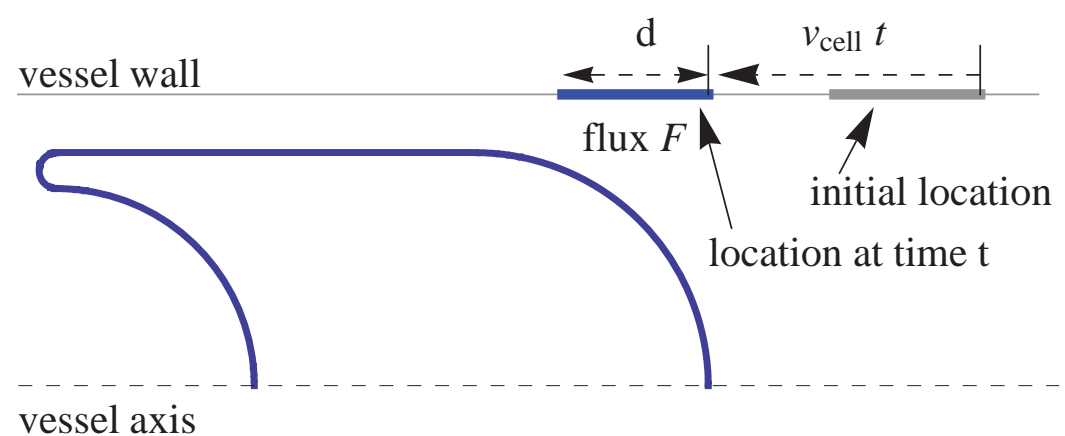

Fig. (4). Time-dependent boundary condition. In the moving reference frame, the cells have fixed locations and the vessel wall moves backwards with speed $v_{\text {cell }}$. The boundary condition for the chemical on the vessel wall specifies the flux, which is zero everywhere except along along a small segment of length $d$ which, at time $t$, has moved a distance $v_{\text {cell }} t$ from its initial location. Along this segment the flux $F$ depends on the scenario. In the first scenario, the segment is the sensor, with $d=\ell$ and flux $F=$ kc into the sensor. In the second scenario, the segment is the chemical source, with $d=\lambda$ and flux $F=K$ into the vessel. 
flux to the sensor is nearly periodic in time: in each time interval $L / v_{\text {cell }}$ the cells move along the vessel by one cell spacing, i.e, distance $L$, relative to the sensor band. After this shift, the geometry, fluid flow, concentration and hence the flux to the sensor are the same as before.

Fig. (5) shows how the sensor band absorbs the chemical primarily from the layer of fluid near the wall. Supplementary file 1 is an animated version of this figure. Comparing the different flow speeds and diffusion coefficients in Table 4 shows the sensor captures more chemical at faster speeds, though a decreasing fraction of the total inlet flux, which grows linearly with the fluid speed. The values in the two models, with and without cells, are comparable, indicating the simpler "empty vessel" model is a reasonable approximation.

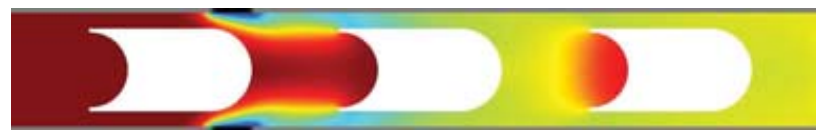

Fig. (5). Sensor on the vessel wall. Concentration around three cells passing the sensor band on the vessel wall. Geometry is as in Fig. (3). Concentration ranges from 0 (dark blue) to $10^{17}$ molecule $/ \mathrm{m}^{3}$ (red). The black lines along the vessel wall mark the location of the sensor at this time, with length $\ell=2 \mu \mathrm{m}$. The cells and fluid move from left to right. Cell speed is $v_{\text {cell }}=1 \mathrm{~mm} / \mathrm{s}$ and diffusion coefficient is $D_{\text {large }}$.

Table 4. Behavior of the Sensor Band on the Vessel Wall

\begin{tabular}{|l|ccc|}
\hline cell speed $(\mathrm{mm} / \mathrm{s})$ & 0.2 & 1 & 2 \\
\hline maximum force $(\mathrm{pN})$ & 10.2 & 51 & 102 \\
$\ldots$ without cells & 10.0 & 50 & 100 \\
\hline \multicolumn{4}{|c|}{ large molecules } \\
average count rate $\left(\mathrm{s}^{-1}\right)$ & 260 & 570 & 800 \\
$\ldots$ without cells & 310 & 590 & 760 \\
\hline & small molecules & & \\
average count rate $\left(\mathrm{s}^{-1}\right)$ & 490 & 1700 & 3700 \\
$\ldots$ without cells & 560 & 2500 & 4200 \\
\hline
\end{tabular}

For comparison, an absorbing sphere of radius $r$ in a region with a chemical with diffusion coefficient $D$ and concentration $C$ far from the sphere, absorbs the chemical at a rate [38].

$4 \pi D a C$

Thus a sphere whose surface area is the same as that of the sensor band, i.e., $2 \pi R \ell$, absorbs at a rate 200 molecule/s when the chemical has diffusion coefficient $D_{\text {large }}$ and concentration $C$ of Table 3 .

For sensors attached to the vessel wall, an important question is the force required to remain attached to the wall. Fig. (6) shows how the shear force on the sensor band varies as cells pass, and compares with the force in the corresponding empty vessel model. In the empty vessel, the flow in a pipe of radius $R$ gives shear force per unit area on the wall of $4 v \eta / R$ where $v$ is the average speed along the vessel, which we take equal to $v_{\text {cell }}$ for this comparison. Multiplying by the surface area of the sensor band, $2 \pi R \ell$, gives the force on the sensor band in the empty vessel. We see the forces in the two models are comparable, so the empty vessel model gives a useful guideline. Moreover, as seen in Fig. (6) the force varies noticeably, by about $25 \%$, as the cell passes, which could be detected with sensors for fluid shear [40].

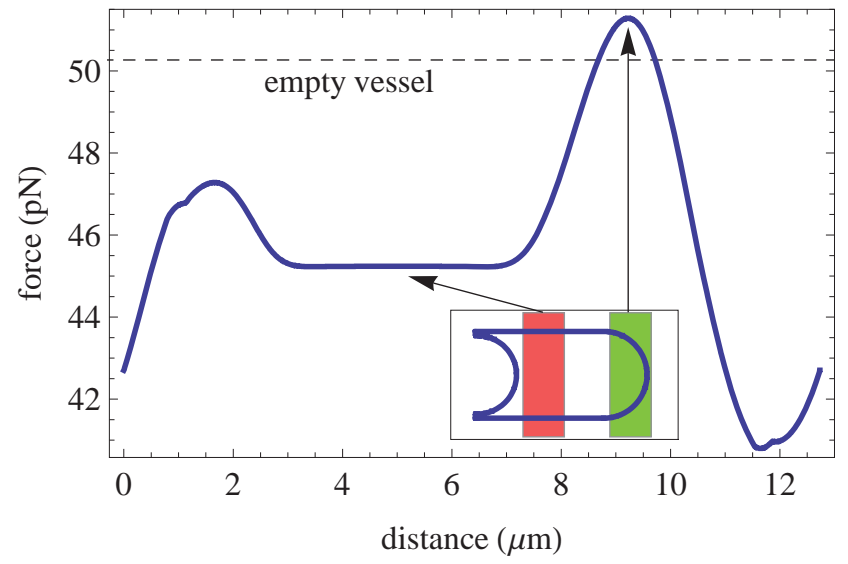

Fig. (6). Shear force, in piconewtons, on the sensor band as a function of its position with respect to a passing cell for $v_{\text {cell }}$ $=1 \mathrm{~mm} / \mathrm{s}$. The dashed line shows the force in the empty vessel model. The inset shows the position of the sensor band with respect to the passing cell at two points on the plot, indicated by the arrows. The total distance shown along the $x$-axis in the figure corresponds to the cell spacing $L$ of Table $\mathbf{1}$.

\section{Sensor Moving with the Fluid}

For the chemical source on the vessel wall, the fluid flow pushes the concentration downstream from the source. Thus the sensor encounters no flux until it reaches and passes the source. As the sensor moves far downstream of the source it continues to encounter the chemical, diluted throughout the vessel, that was released by the source while it was far downstream of the sensor.

Fig. (7) shows one example of how the cells trap the chemical near the vessel wall for a short distance when the diffusion constant is relatively small. However, the subsequent fluid flow and diffusion bring significant flux to the sensor. Supplementary file 2 is an animated version of this figure. Fig. (8) shows how the flux to the sensor varies with its distance past the source for the low and high diffusion cases. Low diffusion, i.e., for large molecules,

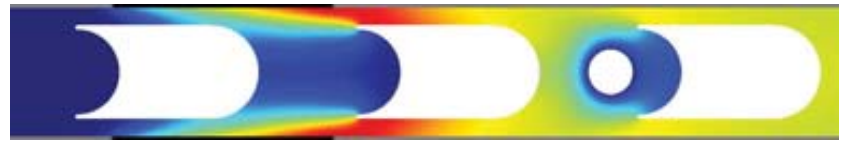

Fig. (7). Sensor moving with the fluid. Concentration around three cells and the sensor shortly after it passes the chemical source. Geometry is as in Fig. (3) with the addition of the sensor, indicated as the small white circle between the center and rightmost cells. Concentration ranges from 0 (dark blue) to $2.5 \times 10^{17}$ molecule $/ \mathrm{m}^{3}$ (red). The black lines along the vessel wall mark the location of the source at this time, with length $\lambda=10 \mu \mathrm{m}$. The cells, sensor and fluid move from left to right. Cell speed is $v_{\text {cell }}=1 \mathrm{~mm} / \mathrm{s}$ and diffusion coefficient is $D_{\text {large }}$. 


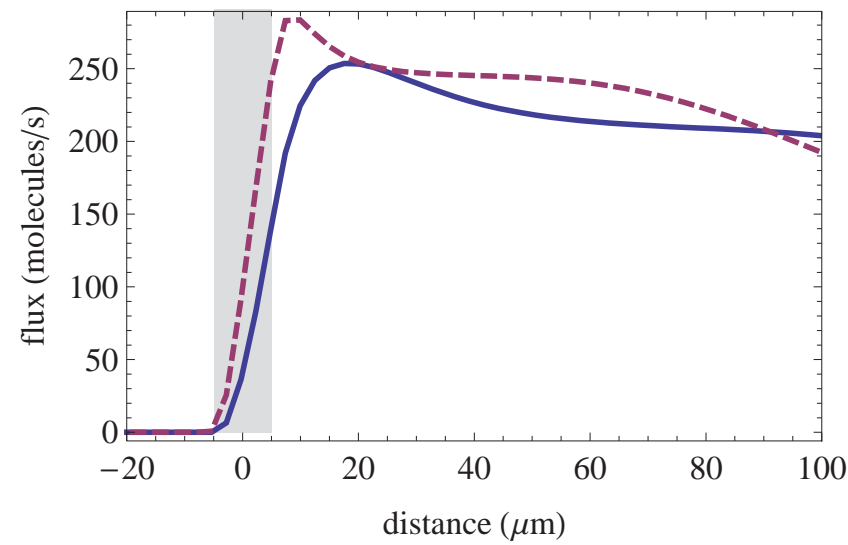

(a)

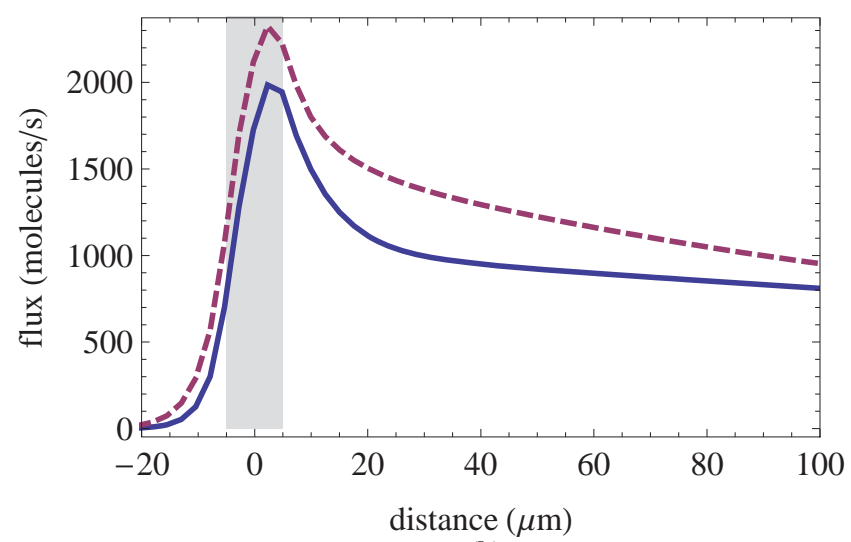

(b)

Fig. (8). Flux to the sensor as a function of distance the center of the sensor is downstream of the center of the source. The solid and dashed curves are with and without cells in the vessel, respectively. (a) large molecules ( $D_{\text {large }}$ diffusion coefficient); (b) small molecules $\left(D_{\text {small }}\right.$ diffusion coefficient). The gray bands show the range of distances where the center of the sensor overlaps with the source, i.e., where the sensor could reach the source by moving radially to the vessel wall.

leads to detection further downstream of the sensor. Fig. (8) also compares the behavior with the model without the cells. The model without cells somewhat overestimates the flux to the sensor, with a maximum closer to the source when diffusion constant is small. These differences are rather modest, indicating the simpler model is adequate for estimating sensing behavior.

Two quantitative summary measures of sensor performance are the maximum rate at which the sensor absorbs molecules at it passes the source, and the number absorbed while the sensor is relatively near the source. As an example for the latter measure, we give the value when the center of the sensor is within $20 \mu \mathrm{m}$ of the source, i.e., for distances in Fig. (8) ranging from -25 to $+25 \mu \mathrm{m}$. The counts while the sensor is near the source indicate how well the sensor can detect the source while it is still relatively nearby. Table 5 gives these values for the different cell speeds and chemical diffusion coefficients, comparing with and without cells in the vessel. The values differ by only about 20\%, indicating models based on empty vessels capture the main behavior of the sensors. Increasing flow

Table 5. Behavior of the Spherical Sensor Moving with the Fluid Past the Chemical Source on the vEssel Wall

\begin{tabular}{|l|ccc|}
\hline cell speed $(\mathrm{mm} / \mathrm{s})$ & 0.2 & 1 & 2 \\
\hline \multicolumn{3}{|c|}{ large molecules } \\
maximum count rate $\left(\mathrm{s}^{-1}\right)$ & 900 & 250 & 130 \\
$\ldots$ without cells & 1000 & 280 & 150 \\
counts while near source & 25 & 5.2 & 2.7 \\
$\ldots$ without cells & 33 & 6.4 & 3.3 \\
\hline & small molecules & & \\
maximum count rate $\left(\mathrm{s}^{-1}\right)$ & 3000 & 2000 & 1500 \\
$\ldots$ without cells & 3500 & 2300 & 1600 \\
counts while near source & 140 & 45 & 27 \\
$\ldots$ without cells & 170 & 58 & 33 \\
\hline
\end{tabular}

speed reduces the counts for the sensor, both because the sensor moves through the high-concentration region near the source more rapidly and also because the count rate itself decreases. Thus at higher speeds, more of the chemical moves downstream past the sensor. This is less of an issue for small molecules, with higher diffusion coefficients.

For comparison, the flux produced by the source is $2 \pi R \lambda K \approx 1900$ molecule/s. This is an upper bound on the steady-state flux to the sensor, i.e., if the sensor captures all the chemical produced by the source. The actual values of Table 5 are well below this rate, except for short periods of time with the lower speeds and high diffusion coefficient.

As another comparison, Eq. (2) gives the flux to a sphere of radius $\ell / 2$ for a chemical with diffusion coefficient $D_{\text {large }}$ and concentration far from the sphere equal to the initial downstream concentration for $v_{\text {cell }}=1 \mathrm{~mm} / \mathrm{s}$ as 100 molecule/s.

\section{DISCUSSION}

In summary, for the two sensing scenarios examined, the simpler model of a vessel without cells gives similar performance estimates as the model with the cells. Neither the change in fluid flow nor confinement of the chemical significantly alter sensor performance. Thus the simpler model is sufficient for design studies of microscopic chemical sensors operating within capillaries. Such studies are relevant both for diagnostics, where the devices attempt to detect specific chemicals, and for power generation where the devices use chemicals in blood plasma as a power source. More broadly, medical applications of these devices involve a variety of performance issues, including sensing, communication, power, and treatments, both individually and in aggregates [6]. Quantitative evaluation of the systemlevel performance requires modeling physical interactions of large numbers of the devices. The results of this paper indicate the modeling of transport within vessels can be simplified by ignoring detailed behavior of the cells while maintaining reasonable accuracy. This simplification greatly extends the computationally feasible range of modeling questions that can be addressed. 
On the other hand, the model with the cells allows evaluating additional aspects of the robots' environment related to the nearby cells. In particular, the variation in flux and forces with the cells show the sensor band on the vessel wall could estimate the rate of passing cells from variations in the counts as well as changes in the fluid shear on devices equipped with flow sensors [40]. From such measurements, the devices could estimate the local hematocrit of the vessel.

The model considers red blood cells of uniform size and spacing. This approach ignores other components of blood, particularly the smaller platelets and the much larger white blood cells, which are much less common than red cells. The model also does not include variation in size and spacing of the red cells, changes in vessel diameter and effects on the flow at branching vessels. Moreover, the distorted cell shapes and vessel walls are taken to be rigid, so the model does not account for any additional changes in cell shape or distortions of the vessel wall as cells move. The model for the first scenario treats the sensor band as being flush with the vessel wall. If instead the sensors extend significantly into the vessel, they will alter the flow and cell shapes. The sensors will experience an additional force due to the pressure difference between their upstream and downstream edges, in both the models, i.e., with and without cells.

As a caveat in interpreting these results, this model is a continuum approximation to a discrete process of individual molecule detections. For macroscopic systems, the number of molecules involved is so large that this approximation accurately represents the detection rates. However, for microscopic sensors, and particularly at low concentrations, the number of molecules involved is relatively small and statistical fluctuations in the actual count become significant (even assuming the sensor itself makes no errors in capturing or identifying the molecules that reach it via diffusion). The continuum approximation gives the average rate of a Poisson process for the counts, with fluctuations proportional to the square root of the number. These fluctuations are a significant source of noise for microscopic sensors, and limit their ability to discriminate chemical sources of interest from background concentration from other sources [15].

Moreover, we consider the chemical as arising only from the source of interest. In practice, there will be background concentrations of the chemical and the sensing task will need to distinguish high concentrations over small distances from a lower but pervasive background concentration. Thus the sensors' detection algorithm must handle statistical fluctuations from the background concentration occasionally appearing to indicate a high localized concentration [15].

Extensions of this model of microscopic devices interacting with cells in capillaries could address other scenarios. Examples include chemicals released by the red blood cells (such as oxygen), branching and changes in size of vessel in which the numerical method must include changes to cell shape [37] and forces on the cells. The last case is relevant for safety by evaluating whether the force groups of sensors impose on passing cells could damage the cells. The model is also relevant for drug delivery tasks targeted to the bloodstream or its components, and could include diffusion of these chemicals out of the capillary to surrounding tissues [23]. Another scenario is the detection of a transient chemical source rather than a steady-state sources considered here. In this case, the time history of the transient may be of interest. The model including cells could determine how cells in the fluid alter that history as observed by the sensor as an additional check on the adequacy of the simpler model without cells.

More generally, this work illustrates how models originally developed to study transport properties in small vessels extend to engineering design studies of the behaviors and environments of microscopic robots. By characterizing chemical detection rates and locations, these models provide constraints on the performance of individual devices and suggestions for control strategies to enable robust, rapid task performance of medical tasks by large groups of robots.

\section{ACKNOWLEDGEMENTS}

I thank R. Freitas Jr. for helpful discussions.

\section{SUPPLEMENTARY MATERIAL}

This article is also acompained by supplementary material and it can be viewed at www.bentham.org/open/ tomnj.

\section{REFERENCES}

[1] Morris K. Macrodoctor, come meet the nanodoctors. The Lancet 2001; 10; 357: 778 .

[2] NIH. National Institutes of Health Roadmap: Nanomedicine; 2003. Available at nihroadmap.nih.gov/nanomedicine/index.asp.

[3] Keszler BL, Majoros IJ, Baker JR. Molecular Engineering in nanotechnology: structure and composition of multifunctional devices for medical application. In Proc. of the Ninth Foresight Conference on Molecular Nanotechnology; 2001

[4] Vo-Dinh T, Kasili P, Wabuyele M. Nanoprobes and Nanobiosensors for monitoring and imaging individual living cells. nanomedicine: Nanotechnology, Biology, and Medicine 2006; 2: 22-30.

[5] Allen TM, Cullis PR. Drug delivery systems: Entering the mainstream. Science 2004; 303: 1818-22.

[6] Freitas RA Jr. Nanomedicine. vol. I: Basic capabilities. georgetown, TX: Landes Bioscience, 1999; Available at www.nanomedicine.com/NMI.htm.

[7] Barreiro A, Rurali R, Hernández ER, Moser J, Pichler T, Forró L, Bachtold A. Subnanometer motion of cargos driven by thermal gradients along carbon nanotubes. Science 2008; 320: 775-8.

[8] Collier CP, Wong EW, Belohradsky M, Raymo FM, Stoddart JF, Kuekes PJ, Williams RS, Heath JR. Electronically configurable molecular-based logic gates. Science 1999; 285: 391-4.

[9] Craighead HG. Nanoelectromechanical systems. Science 2000; 290: 1532-1535.

[10] Howard J. Molecular motors: Structural adaptations to cellular functions. Nature 1997; 389: 561-7.

[11] Fritz J, Baller MK, Lang HP, Rothuizen H, Vettiger P, Meyer E Güntherodt, Gerber C, Gimzewski JK. Translating biomolecular recognition into nanomechanics. Science 2000; 288: 316-8.

[12] Montemagno C, Bach and G. constructing nanomechanical devices powered by biomolecular motors. Nanotechnology 1999; 10: 22531.

[13] In Wang SY, Williams RS, eds. Special issue of Applied Physics A Nanoelectronics. vol. 80. New York: Springer; 2005.

[14] Berg HC, Purcell EM. Physics of chemoreception. Biophys J 1977; 20: 193-219.

[15] Hogg T, Kuekes PJ. Mobile microscopic sensors for highresolution in vivo diagnostics. Nanomedicine: Nanotechnology, Biology, and Medicine 2006; 2: 239-47.

[16] Li H, Tan J, Zhang M. Dynamics modeling and analysis of a swimming microrobot for controlled drug delivery. In Proc. of the IEEE Intl. Conf. on Robotics and Automation (ICRA2006); 2006. p. 1768-73. 
[17] Freitas Jr RA. Pharmacytes: An ideal vehicle for targeted drug delivery. J Nanosci Nanotechnol 2006; 6: 2769-75.

[18] Tucker R, Katira P, Hess H. Hearding nanotransporters: Localized Activation via release and sequestration of control molecules. Nano Letters 2008; 8: 221-6.

[19] Sretavan D, Chang W, Keller C, Kliot M. Microscale surgery on axons for nerve injury treatment. Neurosurgery 2005; 57(4): 63546.

[20] Hogg T, Sretavan DW. Controlling tiny multi-scale robots for nerve repair. In: Proc. of the 20th Natl. Conf. on Artificial Intelligence (AAAI2005). AAAI Press; 2005. p. 1286-91.

[21] Chaudhuri SK, Lovley DR. Electricity generation by direct oxidation of glucose in mediatorless microbial fuel cells. Nat Biotechnol 2003; 21: 1229- 32 .

[22] Keller KH. Effect of fluid shear on mass transport in flowing blood. In: Proc. of Federation of American Societies for Experimental Biology; 1971. p. 1591-9.

[23] Popel AS. Theory of oxygen transport to tissue. Crit Rev Biomed Eng 1989; 17: 257-321.

[24] Krogh A. The number and distribution of capillaries in muscles with calculations of the oxygen pressure head necessary for supplying the tissue. J Physiol 1919; 52: 409-15.

[25] Squires TM, Quake SR. Microfluidics: Fluid physics at the nanoliter scale. Rev Modern Phys 2005; 77: 977-1026.

[26] Purcell EM. Life at low reynolds number. Am J Phys 1977; 45: 311 .

[27] Vogel S. Life in moving fluids. 2nd ed. Princeton Univ. Press, 1994.
[28] Secomb TW, Hsu R, Pries AR. Motion of red blood cells in a capillary with an endothelial surface layer: effect of flow velocity. Am J Physiol: Heart and Circ Physiol 2001; 281: H629-36.

[29] Pozrikidis C. Axisymmetric motion of a file of red blood cells through capillaries. Phys Fluids 2005; 17: 031503.

[30] Pries AR, Secomb TW, Gaehtgens P. Biophysical aspects of blood flow in the microvasculature. Cardiovasc Res 1996; 32: 654-67.

[31] Boryczko K, Dzwinel W, Yuen DA. Dynamical clustering of red blood cells in capillary vessels. J Mol Model 2003; 9: 16-33.

[32] Vadapalli A, Goldman D, Popel AS. Calculations of oxygen transport by red blood cells and hemoglobin solutions in capillaries. Artificial Cells, Blood Subst Immobil Biotechnol 2002; 30: 157-88.

[33] Hogg T. Coordinating Microscopic Robots in viscous fluids. Auton Agents Multi-Agent Syst 2007; 14: 271-305.

[34] Service RF. Proteomics ponders prime time. Science 2008; 321: $1758-61$.

[35] Comsol Multiphysics; 2008. comsol.com.

[36] Karniadakis G, Beskok A, Aluru N. Microflows and nanoflows: fundamentals and simulation. Berlin: Springer; 2005.

[37] Mauroy B. Following red blood cells in a pulmonary capillary, 2007.

[38] Berg HC. Random walks in biology. 2nd ed. Princeton Univ. Press, 1993.

[39] Sheehan PE, Whitman LJ. Detection limits for nanoscale biosensors. Nanoletters 2005; 5(4): 803-7.

[40] Ghosh S, et al. Carbon nanotube flow sensors. Science 2003; 299: $1042-4$. 